\title{
Impacts of biological heating and degradation during bale storage on the surface properties of corn stover
}

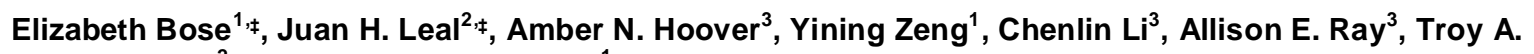
Semelsberger $^{2, *}$, and Bryon S. Donohoe ${ }^{1, *}$

${ }^{1}$ Bioenergy Center, National Renewable Energy Laboratory (NREL)

15013 Denver West Parkway, Golden, CO, 80401, USA;

${ }^{2}$ Material Physics Applications Division, Los Alamos National Laboratory,

P.O. Box 1663, Los Alamos, NM, 87545, USA;

${ }^{3}$ Energy \& Environment Science \& Technology, Idaho National Laboratory,

1955 N. Fremont Avenue, Idaho Falls, ID, 83415, USA

$\ddagger$ Equal contributors

${ }^{*}$ Corresponding authors:

troy@lanl.gov

bryon.donohoe@nrel.gov

\section{Keywords}

biomass feedstock, surface characterization, corn stover, biological degradation, surface area, porosity

\section{Supporting information}

Pages S1-S4

Table S1. Median and interquartile values of $\mathrm{Rq}$, surface area, and fractal dimension calculated from stereomicrographs.

Table S2. Summary of results of Kruskal-Wallis rank sum test on texture results from stereomicrographs.

Table S3. Median and interquartile values of $\mathrm{Rq}$, surface area, and fractal dimension calculated from SEM micrographs.

Table S4. Summary of results of Kruskal-Wallis rank sum test on texture results from SEM micrographs. 


\begin{tabular}{|c|c|c|c|c|}
\hline Table S1 & & \multicolumn{3}{|c|}{ Median (IQR) } \\
\hline $\begin{array}{l}\text { Anatomical } \\
\text { fraction }\end{array}$ & $\begin{array}{l}\text { Level of } \\
\text { degradation }\end{array}$ & $R q(g s v)$ & $\mathrm{SA}\left(\mathrm{mm}^{2}\right)$ & $\mathrm{F}_{\mathrm{D}}$ \\
\hline \multirow{3}{*}{ Leaf top } & Mild & $27.89(25.74,30.05)$ & $1.69(1.57,1.81)$ & $2.8114(2.80215,2.82065)$ \\
\hline & Moderate & $33.97(29.53,38.41)$ & $1.94(1.75,2.13)$ & $2.8238(2.8050,2.8426)$ \\
\hline & Severe & $31.94(28.75,35.12)$ & $1.86(1.72,2)$ & $2.8223(2.8132,2.8315)$ \\
\hline \multirow{3}{*}{$\begin{array}{l}\text { Leaf } \\
\text { bottom }\end{array}$} & Mild & $26.84(23.36,30.31)$ & $1.92(1.73,2.1)$ & $2.8247(2.8017,2.8476)$ \\
\hline & Moderate & $24.93(21.79,28.07)$ & $1.93(1.78,2.09)$ & $2.8345(2.8166,2.8525)$ \\
\hline & Severe & $26.25(22.32,30.18)$ & $1.96(1.85,2.07)$ & $2.8239(2.8028,2.8450)$ \\
\hline \multirow{3}{*}{$\begin{array}{l}\text { Stalk } \\
\text { exterior }\end{array}$} & Mild & $34.20(29.64,38.77)$ & $2.02(1.86,2.18)$ & $2.8415(2.8306,2.8524)$ \\
\hline & Moderate & $35.26(32.56,37.96)$ & $2.18(1.81,2.56)$ & $2.8444(2.8270,2.8617)$ \\
\hline & Severe & $29.17(24.12,34.21)$ & $1.98(1.71,2.24)$ & $2.8313(2.8092,2.8534)$ \\
\hline \multirow{3}{*}{$\begin{array}{l}\text { Stalk } \\
\text { interior }\end{array}$} & Mild & $21.82(19.91,23.72)$ & $1.65(1.51,1.8)$ & $2.8121(2.7823,2.8418)$ \\
\hline & Moderate & $20.89(18.75,23.03)$ & $1.72(1.61,1.83)$ & $2.8172(2.801,2.8333)$ \\
\hline & Severe & $25.74(23.22,28.26)$ & $2.09(1.89,2.29)$ & $2.8386(2.8219,2.8552)$ \\
\hline
\end{tabular}

Table S1. Median and interquartile values of Rq, surface area, and fractal dimension calculated from stereomicrographs.

\begin{tabular}{|c|c|c|c|c|}
\hline \multirow{2}{*}{ Table S2 } & $\begin{array}{c}\text { Anatomical } \\
\text { fraction }\end{array}$ & $\begin{array}{c}\text { Kruskal- } \\
\text { Wallis chi- } \\
\text { squared }\end{array}$ & df & p-value \\
\hline \multirow{3}{*}{ Rq } & Leaf top & 10.694 & 2 & 0.004762 \\
\cline { 2 - 5 } & Leaf bottom & 0.51871 & 2 & 0.7715 \\
\cline { 2 - 5 } & Stalk exterior & 4.1368 & 2 & 0.1264 \\
\cline { 2 - 5 } & Stalk interior & 16.114 & 2 & 0.0003169 \\
\hline \multirow{3}{*}{ SA } & Leaf top & 10.64 & 2 & 0.004893 \\
\cline { 2 - 5 } & Leaf bottom & 0.28903 & 2 & 0.8654 \\
\cline { 2 - 5 } & Stalk exterior & 4.3071 & 2 & 0.1161 \\
\cline { 2 - 5 } & Stalk interior & 19.355 & 2 & $6.27 \mathrm{E}-05$ \\
\hline
\end{tabular}




\begin{tabular}{|c|c|c|c|c|}
\hline \multirow{3}{*}{$\mathrm{Db}$} & Leaf top & 7.1716 & 2 & 0.02771 \\
\cline { 2 - 5 } & Leaf bottom & 1.3548 & 2 & 0.5079 \\
\cline { 2 - 5 } & Stalk exterior & 3.5253 & 2 & 0.1716 \\
\cline { 2 - 5 } & Stalk interior & 10.903 & 2 & 0.004289 \\
\hline
\end{tabular}

Table S2. Summary of results of Kruskal-Wallis rank sum test on texture results from stereomicrographs.

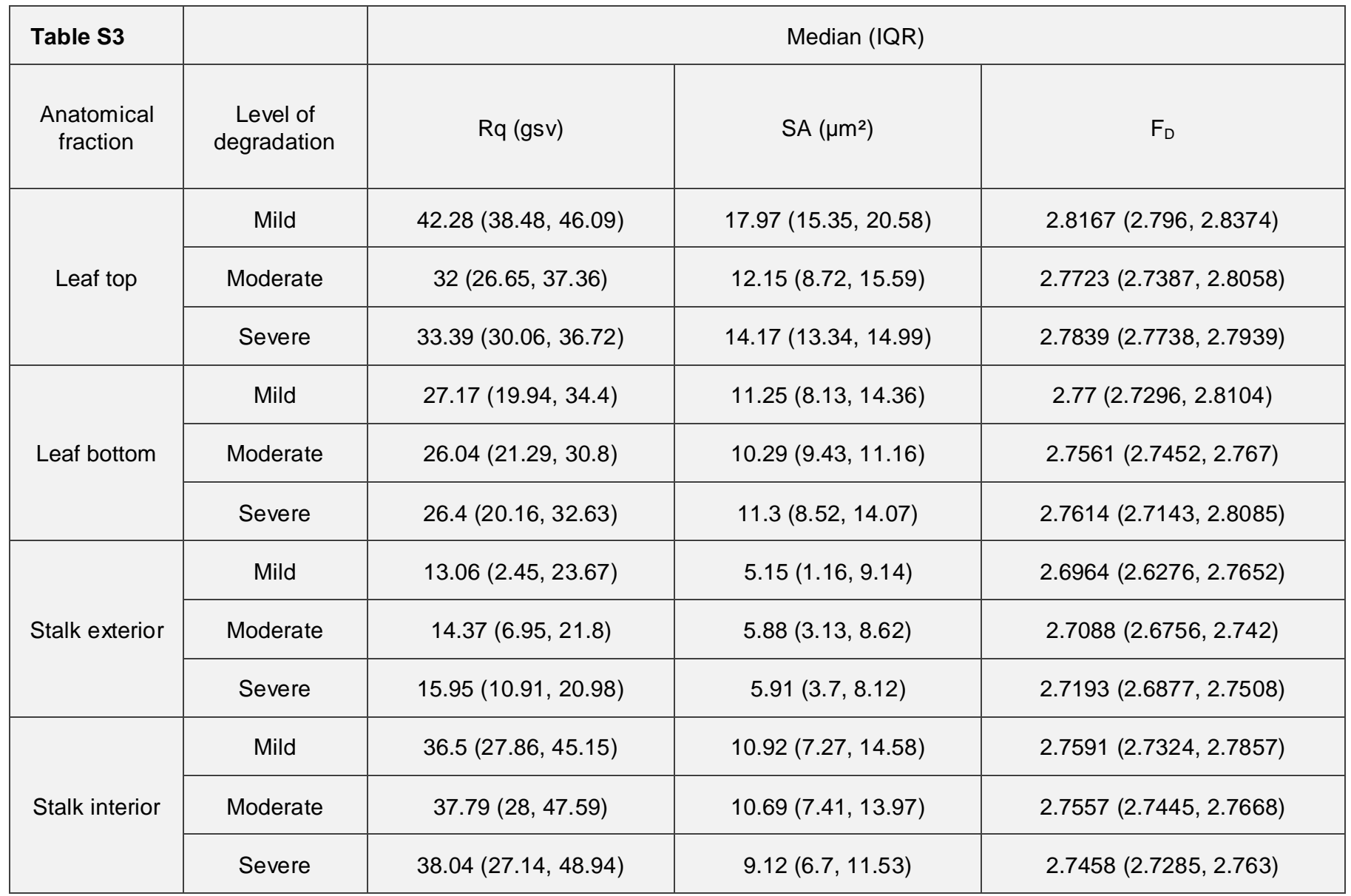

Table S3. Median and interquartile values of Rq, surface area, and fractal dimension calculated from SEM micrographs.

\begin{tabular}{|c|c|c|c|c|}
\hline \multirow{2}{*}{ Table S4 } & $\begin{array}{c}\text { Anatomical } \\
\text { fraction }\end{array}$ & $\begin{array}{c}\text { Kruskal- } \\
\text { Wallis chi- } \\
\text { squared }\end{array}$ & df & p-value \\
\hline \multirow{2}{*}{$\mathrm{Rq}$} & Leaf top & 12.596 & 2 & 0.00184 \\
\cline { 2 - 5 } & Leaf bottom & 0.23484 & 2 & 0.8892 \\
\cline { 2 - 5 } & Stalk exterior & 0.28129 & 2 & 0.8688 \\
\hline
\end{tabular}




\begin{tabular}{|c|c|c|c|c|}
\hline \multirow{3}{*}{ SA } & Stalk interior & 0.28129 & 2 & 0.8688 \\
\hline & Leaf top & 12.48 & 2 & 0.00195 \\
\cline { 2 - 5 } & Leaf bottom & 0.70452 & 2 & 0.7031 \\
\cline { 2 - 5 } & Stalk exterior & 1.28 & 2 & 0.5273 \\
\cline { 2 - 5 } & Stalk interior & 1.969 & 2 & 0.3736 \\
\hline \multirow{3}{*}{$\mathrm{Db}$} & Leaf top & 11.166 & 2 & 0.00376 \\
\cline { 2 - 5 } & Leaf bottom & 1.68 & 2 & 0.4317 \\
\cline { 2 - 5 } & Stalk exterior & 2.3303 & 2 & 0.3119 \\
\cline { 2 - 5 } & Stalk interior & 1.3958 & 2 & 0.4976 \\
\hline
\end{tabular}

Table S4. Summary of results of Kruskal-Wallis rank sum test on texture results from SEM micrographs. 